\title{
Inter-helical and Intra-helical Buckling Analyses of Tubular Strings with Connectors in Horizontal Wellbores
}

\author{
Wenjun Huang ${ }^{a, b}$, Deli Gao ${ }^{b}$, Yinghua Liu ${ }^{a}$ \\ ${ }^{a}$ School of Aerospace Engineering, AML, Tsinghua University, Beijing 100084, China \\ ${ }^{b}$ MOE Key Laboratory of Petroleum Engineering, China University of Petroleum, Beijing 102249, China \\ *Corresponding author: huangwenjun1986@126.com, gaodeli@cast.org.cn, yhliu@tsinghua.edu.cn
}

\begin{abstract}
Helical buckling of tubular strings with connectors is divided into two sub-problems: inter-helical buckling and intra-helical buckling. The suspended section of the tubular string is depicted by beam-column model and the continuous contact section is depicted by helix. Further introducing the continuous conditions, boundary conditions and periodic conditions, the deflection curves of tubular strings can be determined. On the basis of energy stability condition, the critical helical buckling loads are deduced by introducing the potential energy factor. The contact forces on contact points and continuous contact sections and maximum bending moments on tubular strings are calculated. The above studied are further applied into a case study. At last, the supporting effect and boundary effect caused by connectors are introduced and the relationship between inter-helical buckling and intra-helical buckling modes is clarified. The results indicate that for connectors of smaller diameters, the supporting effect plays the most important role and inter-helical buckling is easier to initiate. However, for connectors of larger diameters, the constraint effect is dominant and intra-helical buckling is more likely to occur. Increasing connector diameter and decreasing the length between adjacent connectors can increase critical helical buckling loads. Both the contact forces and maximum bending moments in the intra-helical buckling mode are larger than that in the inter-helical buckling mode, so intra-helical buckling should be avoided in the optimal design of connectors on tubular strings.
\end{abstract}

Keywords: tubular mechanics; helical buckling; connector; contact force; bending moment

\section{Introduction}

Until now, many researchers have conducted a series of studies on the lateral buckling, sinusoidal buckling and helical buckling behaviors of tubular strings constrained in vertical, inclined (horizontal) and curved wellbores with energy method and buckling differential equation (Lubinski 1950, 1962; Paslay 1964; Mitchell 1982, 1988, 1999, 2002; Chen 1990; Gao 1998, 2002, 2006, 2013; Gao 2009, 2010; Miska 1995; Wu 1992, 1995; Huang 2015a, 2015b) and a mature theoretical framework of tubular buckling has been established. In the above studies, the entire tubular string is taken as a uniformly smooth rod and the effects of connectors on tubular strings are neglected. Lubinski (1977) and Paslay (1991) studied the lateral deflection of a weightless tubular string under axial tension and compression constrained in a curved wellbore. Gao (2012) studied the critical lateral buckling condition for a tubular string constrained in a horizontal wellbore. On the basis of Paslay and Gao's results, Huang (2015d) studied the lateral buckling for a tubular string with weight constrained in a curved wellbore. Mitchell $(2000,2003 a, 2003 b, 2006)$ 
studied the helical buckling of the tubular string with connectors in no contact case in vertical wellbores and the sinusoidal buckling in no contact case in inclined and curved wellbores. Huang (2014a; 2014b; 2015c; 2016) extended Mitchell's work and studied the sinusoidal buckling and helical buckling under three contact cases in vertical, horizontal and curved wellbores.

However, the previous mechanical analyses on tubular strings with connectors are not mature, since the critical buckling loads, contact forces and maximum bending moments for different buckling modes have not been deduced. Meanwhile, the coupling effects of connector and buckling are not sufficiently revealed. Especially for the helical buckling problem, connectors have two different kinds of effects and lead to two kinds of helical buckling modes, which have not been concerned in the previous studies. In this paper, the inter-helical buckling and intra-helical buckling problems are studied. The critical buckling loads, contact forces and maximum bending moments are deduced by mechanical analysis. The supporting effect and boundary effect caused by connectors are introduced and the relationship between inter-helical buckling and intra-helical buckling modes is clarified.

\section{Inter-helical buckling of tubular strings with connectors}

Two kinds of helical buckling including inter-helical buckling and intra-helical buckling are discussed. Inter-helical buckling means that the an whole buckling configuration occurs across several adjacent connectors, while intra-helical buckling means that helical buckling only happens on a portion of the tubular string between adjacent connectors. The investigation in these two helical buckling problems is based on the following assumptions:

1. The tubular string is taken as a thin rod and the horizontal wellbore as an ideal horizontal cylinder.

2. The tubular string deflection is a small term with respect to the tubular string length and in elastic range.

3. Connectors are evenly distributed along the tubular string and in contact with the inner surface of the wellbore.

4. Every portion of the tubular string between two adjacent connectors has the same deflection curve.

5. Friction and torque are neglected. 


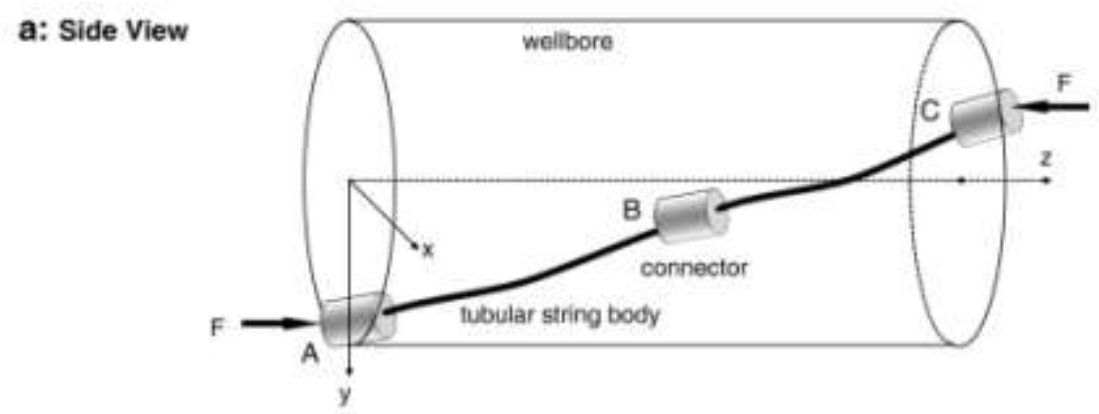

\section{b: Cross Section}

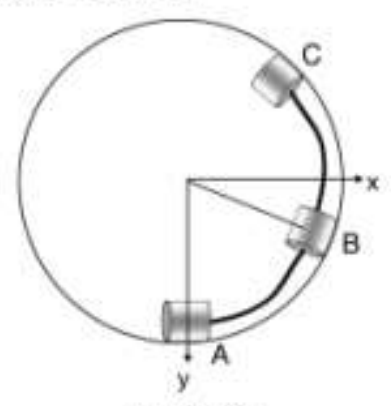

no contact

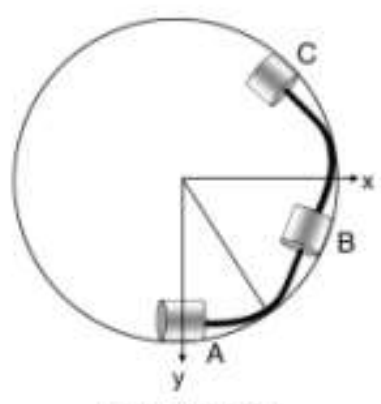

point contact

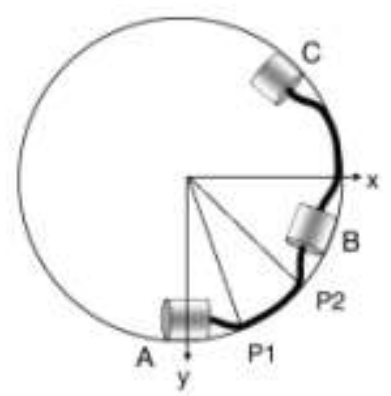

wrap contact

Fig. 1. Schematic of helical buckling of a tubular string with connectors constrained in a horizontal wellbore: (a) no contact (b) point contact (c) wrap contact

\subsection{Critical buckling loads}

Assuming that $\mathrm{x}$ and $\mathrm{y}$ are the lateral displacements along $\mathrm{x}$-axis and $\mathrm{y}$-axis, $\mathrm{z}$ is the axial distance along z-axis shown in Fig. 1 , the potential energy of tubular string caused by axial force and bending moment is calculated by (Gao, 2006)

$$
\begin{aligned}
\Pi_{p} & =\frac{E I}{2} \int_{0}^{L}\left[\left(\frac{\partial^{2} x}{\partial z^{2}}\right)^{2}+\left(\frac{\partial^{2} y}{\partial \eta^{2}}\right)^{2}\right] d z-\frac{F}{2} \int_{0}^{L}\left[\left(\frac{\partial \hat{x}}{\partial \eta}\right)^{2}+\left(\frac{\partial \hat{y}}{\partial \eta}\right)^{2}\right] d z \\
& =\frac{E I r_{c}^{2}}{2 L^{3}}\left\{\int_{0}^{1}\left[\left(\frac{\partial^{2} \hat{x}}{\partial \eta^{2}}\right)^{2}+\left(\frac{\partial^{2} \hat{y}}{\partial \eta^{2}}\right)^{2}\right]-u^{2}\left[\left(\frac{\partial \hat{x}}{\partial \eta}\right)^{2}+\left(\frac{\partial \hat{y}}{\partial \eta}\right)^{2}\right] d \eta\right\} \text { (1) }
\end{aligned}
$$

Where $\eta$ is the dimensionless distance equal to $z / L, L$ is the length of tubular strings between adjacent connectors, $\hat{x}$ and $\hat{y}$ are the dimensionless lateral displacements calculated by $\hat{x}=\frac{x}{r_{c}}$ and $\hat{y}=\frac{y}{r_{c}}, r_{c}$ is the radial clearance between the connector and wellbore, and $\mathrm{u}$ is the dimensionless axial compression defined by $u=\sqrt{\frac{F}{E I}} \cdot L, \mathrm{~F}$ is the axial compression, $\mathrm{El}$ is the bending stiffness of the tubular string.

For the non-connector case, the deflection curve of the tubular string is $\hat{x}=\delta_{b} \sin \left(\frac{u}{\sqrt{2}} \eta\right)$ and $\hat{y}=\cos \delta_{b}\left(\frac{u}{\sqrt{2}} \eta\right)$ (Lubinski, 1962), where the term $\delta_{b}=\frac{r_{b}}{r_{c}}$ is the ratio of the radial clearance between tubular string body and wellbore $\left(r_{b}\right)$ to that between the connectors and wellbore $\left(r_{c}\right)$, Eq. (1) is simplified into 


$$
\Pi_{p}^{0}=\frac{E I r_{c}^{2}}{2 L^{3}} \cdot\left(-\frac{u^{4} \delta_{b}^{2}}{4}\right)(2)
$$

The potential energy factor is defined by the ratio of Eq. (1) to Eq. (2), namely

$$
\lambda_{\pi, p}=\frac{\Pi_{p}}{\Pi_{p}{ }^{0}}=-\frac{4}{u^{4} \delta_{b}^{2}}\left\{\int_{0}^{1}\left[\left(\frac{\partial^{2} \hat{x}}{\partial \eta^{2}}\right)^{2}+\left(\frac{\partial^{2} \hat{y}}{\partial \eta^{2}}\right)^{2}\right]-u^{2}\left[\left(\frac{\partial \hat{x}}{\partial \eta}\right)^{2}+\left(\frac{\partial \hat{y}}{\partial \eta}\right)^{2}\right] d \eta\right\} \text { (3) }
$$

The detailed calculation procedures of deflection curves of tubular strings with connectors under different contact cases are given in Appendix A. The results indicate that the tubular strings go through no contact, point contact and wrap contact with the increase of axial compressions. Substituting the expressions of $\hat{x}$ and $\hat{y}$ into Eq. (3), the value of potential energy factor is determined.

If the potential energy due to tubular gravity is further considered, the total potential energy change is calculated by

$$
\begin{aligned}
\Pi_{t} & =\Pi_{p}+\Pi_{g} \\
& =\frac{E I r_{c}^{2}}{2 L^{3}}\left\{\int_{0}^{1}\left[\left(\frac{\partial^{2} \hat{x}}{\partial \eta^{2}}\right)^{2}+\left(\frac{\partial^{2} \hat{y}}{\partial \eta^{2}}\right)^{2}\right]-u^{2}\left[\left(\frac{\partial \hat{x}}{\partial \eta}\right)^{2}+\left(\frac{\partial \hat{y}}{\partial \eta}\right)^{2}\right] d \eta\right\}+q r_{b} L \\
& =\lambda_{\pi, p} \cdot\left\{\frac{E I r_{b}^{2}}{2 L^{3}} \cdot \int_{0}^{1}\left[\left(\frac{\partial^{2} \hat{x}^{0}}{\partial \eta^{2}}\right)^{2}+\left(\frac{\partial^{2} \hat{y}^{0}}{\partial \eta^{2}}\right)^{2}\right]-\frac{F r_{b}^{2}}{2 L} \cdot\left[\left(\frac{\partial \hat{x}^{0}}{\partial \eta}\right)^{2}+\left(\frac{\partial \hat{y}^{0}}{\partial \eta}\right)^{2}\right] d \eta\right\}+q r_{b} L \\
& =\frac{\left(\lambda_{\pi, p} \cdot E I\right) \cdot r_{b}^{2}}{2 L^{3}} \cdot \int_{0}^{1}\left[\left(\frac{\partial^{2} \hat{x}^{0}}{\partial \eta^{2}}\right)^{2}+\left(\frac{\partial^{2} \hat{y}^{0}}{\partial \eta^{2}}\right)^{2}\right]-\frac{\left(\lambda_{\pi, p} \cdot F\right) \cdot r_{b}^{2}}{2 L} \cdot\left[\left(\frac{\partial \hat{x}^{0}}{\partial \eta}\right)^{2}+\left(\frac{\partial \hat{y}^{0}}{\partial \eta}\right)^{2}\right] d \eta+q r_{b} L
\end{aligned}
$$

In which $\Pi_{g}$ is the potential energy due to tubular gravity, the superscript ${ }^{\circ}$ represents the results in the no connector case. Note that the dimensionless potential energy Eq. (3) is adopted in the derivation of Eq. (4).

For the non-connector case, the total potential energy change is calculated by

$$
\Pi_{t}^{0}=\frac{(1 \cdot E I) \cdot r_{b}^{2}}{2 L^{3}} \cdot \int_{0}^{1}\left[\left(\frac{\partial^{2} \hat{x}^{0}}{\partial \eta^{2}}\right)^{2}+\left(\frac{\partial^{2} \hat{y}^{0}}{\partial \eta^{2}}\right)^{2}\right]-\frac{(1 \cdot F) \cdot r_{b}^{2}}{2 L} \cdot\left[\left(\frac{\partial \hat{x}^{0}}{\partial \eta}\right)^{2}+\left(\frac{\partial \hat{y}^{0}}{\partial \eta}\right)^{2}\right] d \eta+q r_{b} L
$$

By comparing Eq. (4) with (5), the effect of connectors on potential energy can be included by amending the bending stiffness of the tubular string $(E I)$ and the axial force $(F)$ with the factor $\left(\lambda_{\pi, p}\right)$.

The total potential energy change is closely related to the critical helical buckling load. In Chen's (1990) derivation, the critical helical buckling load for non-connector tubular string is obtained by letting $\Pi_{t}^{0}=0$. Here, we extended this principle to the connector case, namely $\Pi_{t}=0$. Considering that Chen's critical helical buckling load is expressed as $F_{h e l}{ }^{0}=\gamma \sqrt{\frac{E I q}{r_{b}}}$, the critical helical buckling load for tubular string with connectors is equal to $\lambda_{\pi, p} \cdot F_{h e l}=\gamma \sqrt{\frac{\lambda_{\pi, p} \cdot E I q}{r_{b}}}$. 
Thus,

$$
\begin{aligned}
& F_{h e l}=\lambda_{\pi, p}^{-\frac{1}{2}} \cdot \gamma \sqrt{\frac{E I q}{r_{b}}}=\lambda_{\pi, p}^{-\frac{1}{2}} \cdot F_{h e l}{ }^{0} \\
& \lambda_{F, h e l}=F_{h e l} / F_{h e l}{ }^{0}=\lambda_{\pi, p}{ }^{-\frac{1}{2}}
\end{aligned}
$$

In which the buckling load factor $\lambda_{F, h e l}$ means the ratio of critical buckling load for the connector case to non-connector case.

Fig. 2 shows the buckling load factor $\left(\lambda_{F, h e l}\right)$ under different values of non-connector buckling load $\left(u_{h e l}{ }^{0}\right)$ and connector parameter $\left(\delta_{b}\right)$. The results show that the critical helical buckling loads for connector case are larger than that for non-connector case, especially in the no contact stage. The buckling load $\left(\lambda_{F, h e l}\right)$ factor decreases with the increase of non-connector buckling load $\left(u_{\text {hel }}{ }^{0}\right)$ and tends to 1 when the non-connector buckling load tends to infinity. With the increase of connector parameter $\left(\delta_{b}\right)$, the buckling load factor also increases.

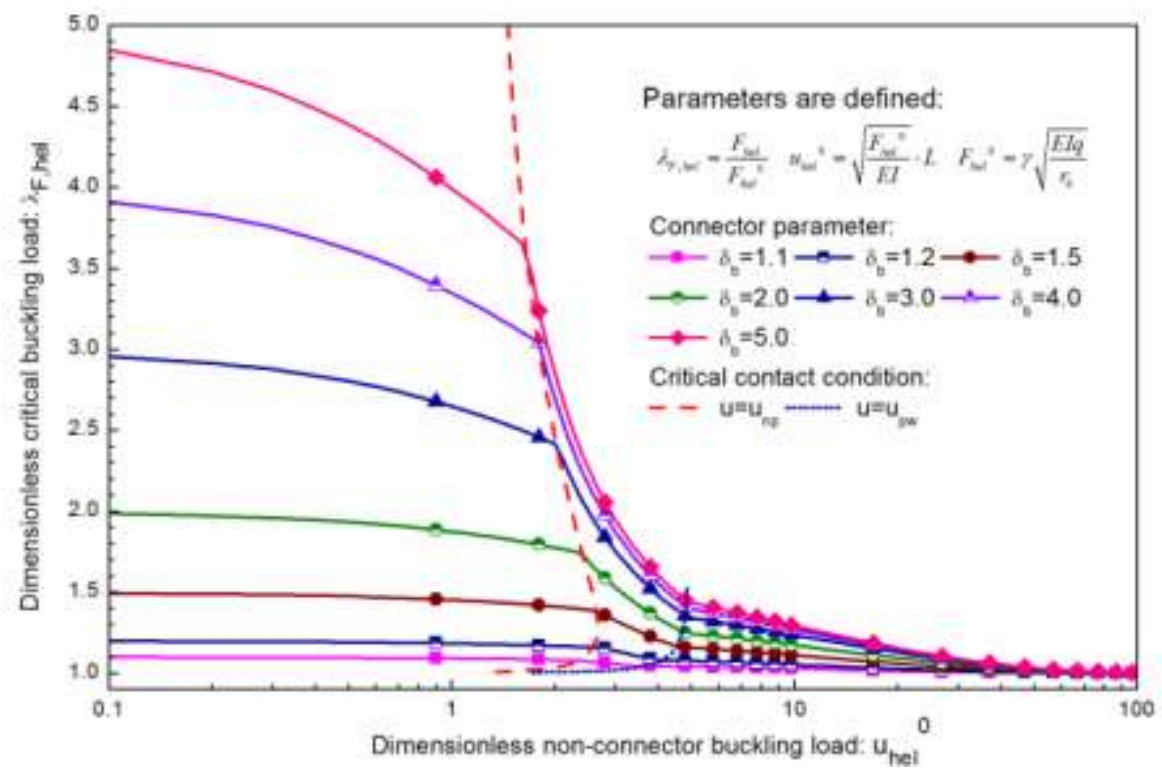

Fig. 2. Critical buckling loads under different values of non-connector buckling load and connector parameter.

\subsection{Contact forces and bending moments}

\section{(1) Contact forces}

The contact force between the tubular string and the wellbore includes that on the connector, contact point and middle continuous contact section. The contact force on the connector is calculated by

$$
N_{c}=-\left.\frac{2 E I r_{c}}{L^{3}} \frac{d^{3} \hat{y}}{d \eta^{3}}\right|_{\eta=0}
$$


The contact force on the contact point is equal to

$$
N_{p}=\lambda_{m} \frac{E I r_{c}}{L^{3}}
$$

The contact force on the continuous contact section is calculated by

$$
n_{w}=\frac{E I r_{\mathrm{c}}}{L^{4}} \frac{\delta_{b} u^{4}}{4}\left(2 \kappa^{2}-\kappa^{4}\right) \cdot \chi L
$$

For the no contact case, the total contact force is equal to the connector contact force, namely $N_{t}=N_{c}$. For the point contact case, the total contact force includes connector and point contact forces, namely $N_{t}=N_{c}+N_{p}$. For the wrap contact case, the total contact force includes connector, point and continuous contact forces, namely $N_{t}=N_{c}+N_{p}+N_{w}$. Here the dimensionless total contact force is defined as the ratio of the contact force $N_{t}$ to non-connector contact force, namely $\lambda_{n, t}=\frac{N_{t}}{N_{t}^{0}}$, which $N_{t}^{0}$ is the total contact force for non-connector tubular string in the helical buckling mode and is equal to $N_{t}^{0}=\frac{r_{b} F^{2}}{4 E I}$. Thus, we obtain

$$
N_{t}=\lambda_{n, t} \cdot \frac{r_{b} F^{2}}{4 E I}
$$

Fig. 3 shows the values of dimensionless contact forces under different axial forces. The results show that the existence of connectors can decrease the total contact force, especially in the no contact stage. The dimensionless contact forces increase with the increase of axial force in the no contact and point contact cases. In the wrap contact case, the dimensionless contact forces firstly decreases and then increases and tends to 1 . With the increase of connector parameter $\left(\delta_{b}\right)$, the total contact force decreases, because the equivalent radial clearance between the tubular string and wellbore is decreased.

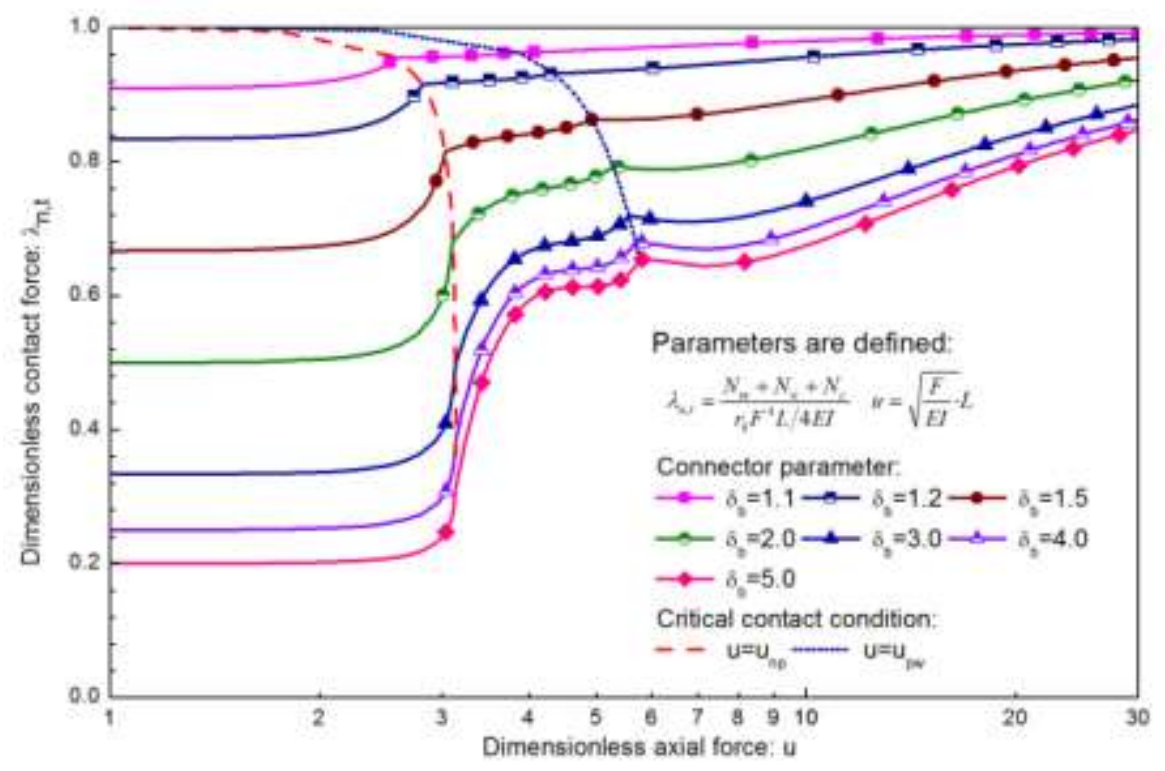

Fig. 3. Dimensionless total contact forces under different axial forces

The ratios of contact forces on connector and tubular string body to the total contact force 
are respectively defined by

$$
\lambda_{n, c}=\frac{N_{c}}{N_{t}} \quad \lambda_{n, b}=1-\frac{N_{c}}{N_{t}}=1-\lambda_{n, c}
$$

Thus, the contact forces on connectors and tubular string bodies are calculated by

$$
N_{c}=\lambda_{n, \mathrm{c}} \cdot \lambda_{n, t} \cdot \frac{r_{b} F^{2}}{4 E I} \quad N_{b}=\lambda_{n, \mathrm{~b}} \cdot \lambda_{n, t} \cdot \frac{r_{b} F^{2}}{4 E I}
$$

Fig. 4 shows the ratios of contact force on connector to the total contact force $\left(\lambda_{n, c}\right)$ under different axial forces $(u)$. In the no contact stage, the connector contact force is equal to the total contact force and the ratio $\left(\lambda_{n, c}\right)$ is equal to 1 . In the point contact force, the ratio $\lambda_{n, c}$ firstly decreases and then increases with the increase of axial force. In the wrap contact case, the ratio $\lambda_{n, c}$ firstly decreases abruptly and then is slowly down to close to 0 . The decrease of the ratio $\lambda_{n, c}$ means that the effect of connectors weakens with the increase of axial force and connectors have no effect when the axial force tends infinity.

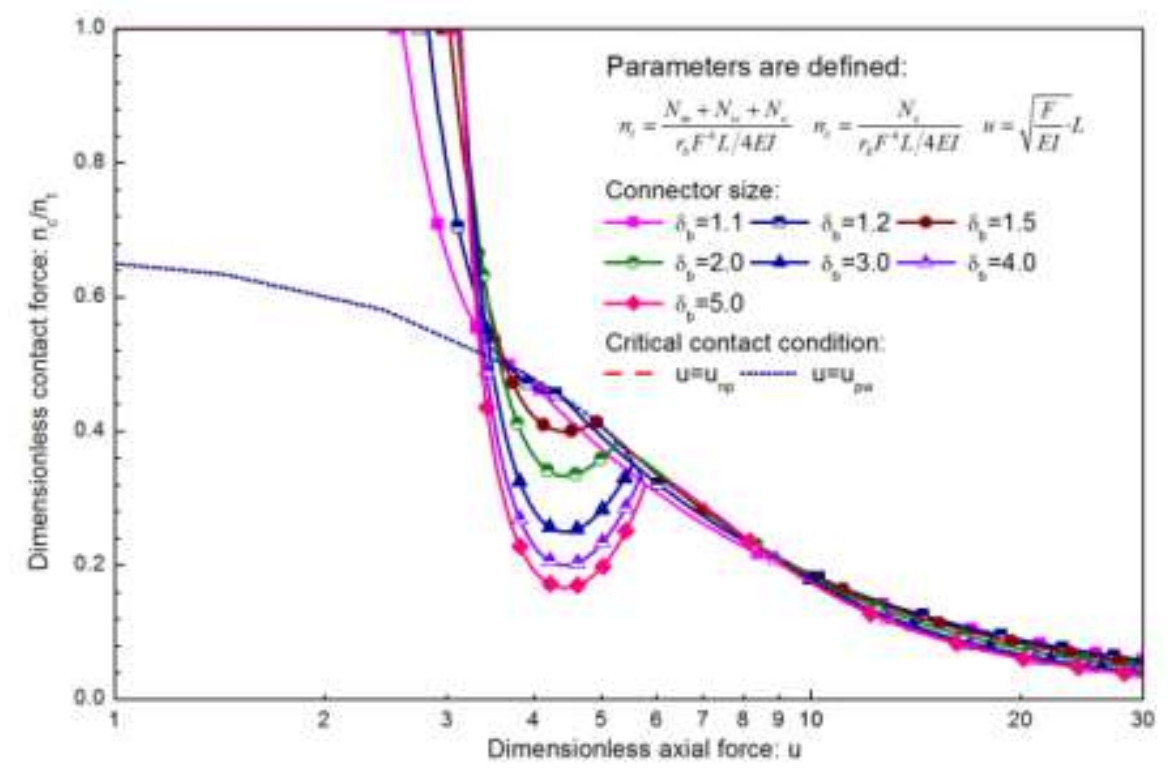

Fig. 4. The ratios of contact force on connector to the total contact force under different axial forces

\section{(2) Bending moments}

The maximum bending moments on the tubular string for the no contact and point contact cases are obtained at the connector or the middle point of the tubular string. For the wrap contact case, the maximum bending moment are obtained on the connector, contact point or wrap contact section of the tubular string. Therefore, the maximum bending moment can be expressed in a general form: 


$$
M_{\max }=\frac{E I r_{c}}{L^{2}} \cdot \max \left(\left.\sqrt{\left(\frac{d^{2} \hat{x}}{d \eta^{2}}\right)^{2}+\left(\frac{d^{2} \hat{y}}{d \eta^{2}}\right)^{2}}\right|_{\eta=0,\left(\frac{1-\chi}{2}\right), \frac{1}{2}}\right)
$$

The dimensionless maximum bending moment is defined as the ratio of Eq. (13) to that of the non-connector tubular string under the first category of boundary condition, namely $m_{\max }=M_{\max } /\left(F r_{b} / 2\right)$. Considering that the maximum bending moment under the secondary category of boundary condition is twice of that under the first category of boundary condition, the maximum bending moment for connector case under the second category of boundary condition are calculated by

$$
M_{\max }=m_{\max } \cdot F r_{b}
$$

Fig. 5 shows the dimensionless maximum bending moments $\left(m_{\max }\right)$ under different axial forces $(u)$. In the no contact stage, the dimensionless maximum bending moment increases with axial force. The maximum value of $m_{\max }$ is achieved at the critical condition from no contact to point contact $\left(u=u_{n p}\right)$. In the point contact stage, the dimensionless maximum bending moment decreases with axial force. In both the no contact and point contact stages, the maximum bending moments are on the middle point of the tubular string. In the wrap contact stage, the value of $m_{\max }$ is increased and tends to 1 with the axial compression.

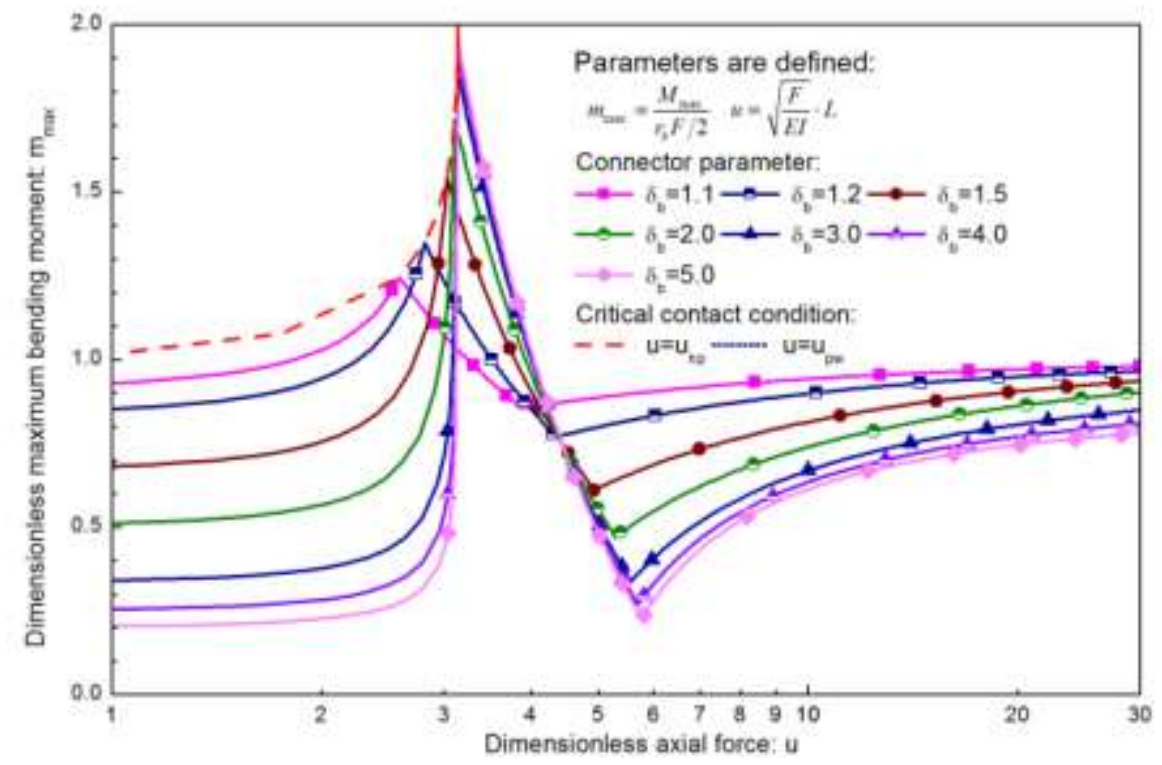

Fig. 5. Dimensionless maximum bending moments under different axial forces

\section{Intra-helical buckling of tubular strings with connectors}

If the diameter of the connectors is close to that of the wellbore, the inter-helical buckling is inhibited and another kind of buckling mode called intra-helical buckling occurs. The investigation of the intra-helical buckling is based on the same assumptions given in inter-helical buckling problem. Here, the boundary conditions on the two adjacent connectors are taken as pinned 
supports.

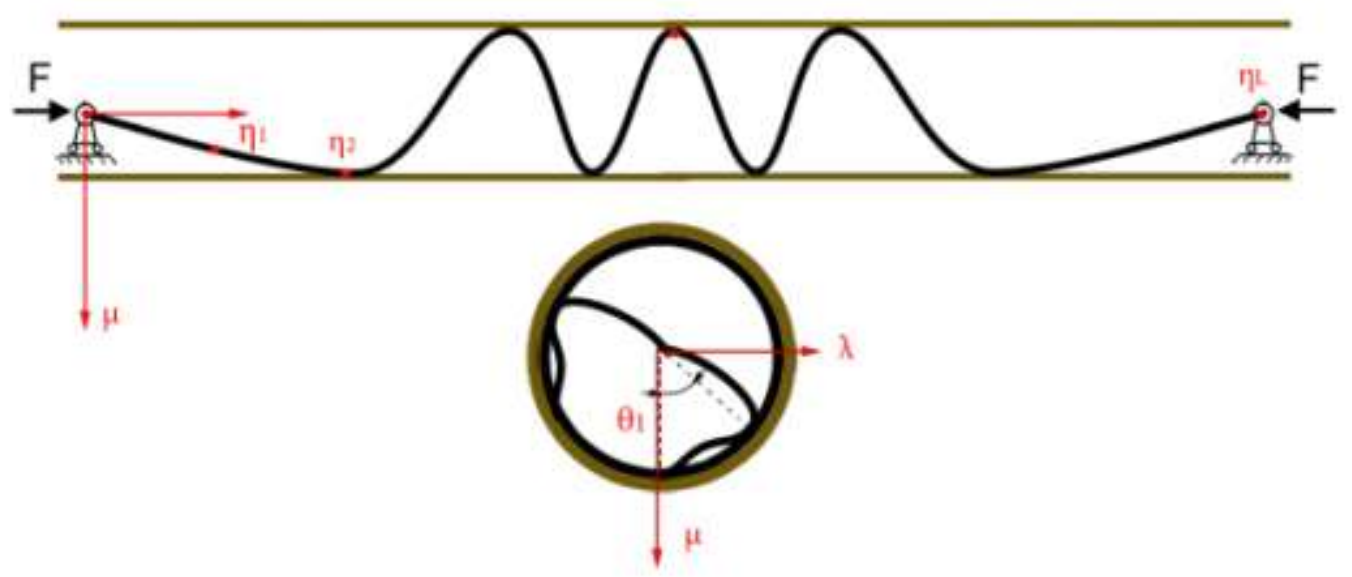

Fig. 6. Schematic of helical buckling of a tubular string with connectors constrained in a horizontal wellbore.

\subsection{Critical buckling loads}

The calculation procedures of intra-helical buckling are given in Appendix B and the results show that the deflection curve of a tubular string is mainly divided into two parts: (1) suspended sections near the boundary supports and (2) continuous contact section in the middle. The suspended sections on each end of the tubular string are further divided into two suspended portions shown in Fig. 6.

Considering that the dimensionless potential energy for intra-helical buckling has the same calculation expression to that for inter-helical buckling, the effect of connector on critical intra-helical buckling load is obtained by introducing the amendment factor of $\lambda_{\pi, \mathrm{p}}$. The value of $\lambda_{\pi, \mathrm{p}}$ can be obtained by substituting the deflection curves of intra-helical buckling given in Appendix B into Eq. (3). Thus, we obtain

$$
\begin{aligned}
& F_{h e l}=\lambda_{\pi, p}{ }^{-\frac{1}{2}} \cdot \gamma \sqrt{\frac{E I q}{r_{b}}}=\lambda_{\pi, p}^{-\frac{1}{2}} \cdot F_{h e l}{ }^{0} \\
& \lambda_{F, h e l}=F_{h e l} / F_{h e l}{ }^{0}=\lambda_{\pi, p}{ }^{-\frac{1}{2}}
\end{aligned}
$$

Under the intra-helical buckling mode, the length of continuous contact section is not smaller than 0 . Thus, one necessary condition for the occurrence of intra-helical buckling according to Appendix $B$ is

$$
F_{h e l} \geq \frac{9.09 \pi^{2} E I}{L^{2}}
$$

Where $E I$ is the bending stiffness of the tubular string and $L$ is the tubular string length between adjacent connectors.

Meanwhile, another necessary condition for intra-helical buckling is that the diameter of connector is close to that of the wellbore, namely 


$$
\delta_{b} \geq \delta_{b, \text { int } r a}
$$

Where $\delta_{b \text {,int } r a}$ is the threshold value for the occurrence of intra-helical buckling and close to 1.

Fig. 7 shows the dimensionless critical buckling loads $\left(\lambda_{F, h e l}\right)$ under different values of non-connector critical buckling loads $\left(\eta_{L, h e l}{ }^{0}\right)$. Note that the critical buckling load $\lambda_{F, h e l}$ means the ratio of critical buckling load in the presence of connectors to that in the non-connector case. The results show that the existence of connectors can increase the critical intra-helical buckling load. The buckling load factor $\left(\lambda_{F, h e l}\right)$ decreases with the increase of non-connector critical buckling load $\left(\eta_{L, h e l}{ }^{0}\right)$ and tends to 1 when the non-connector critical buckling load tends to infinity.

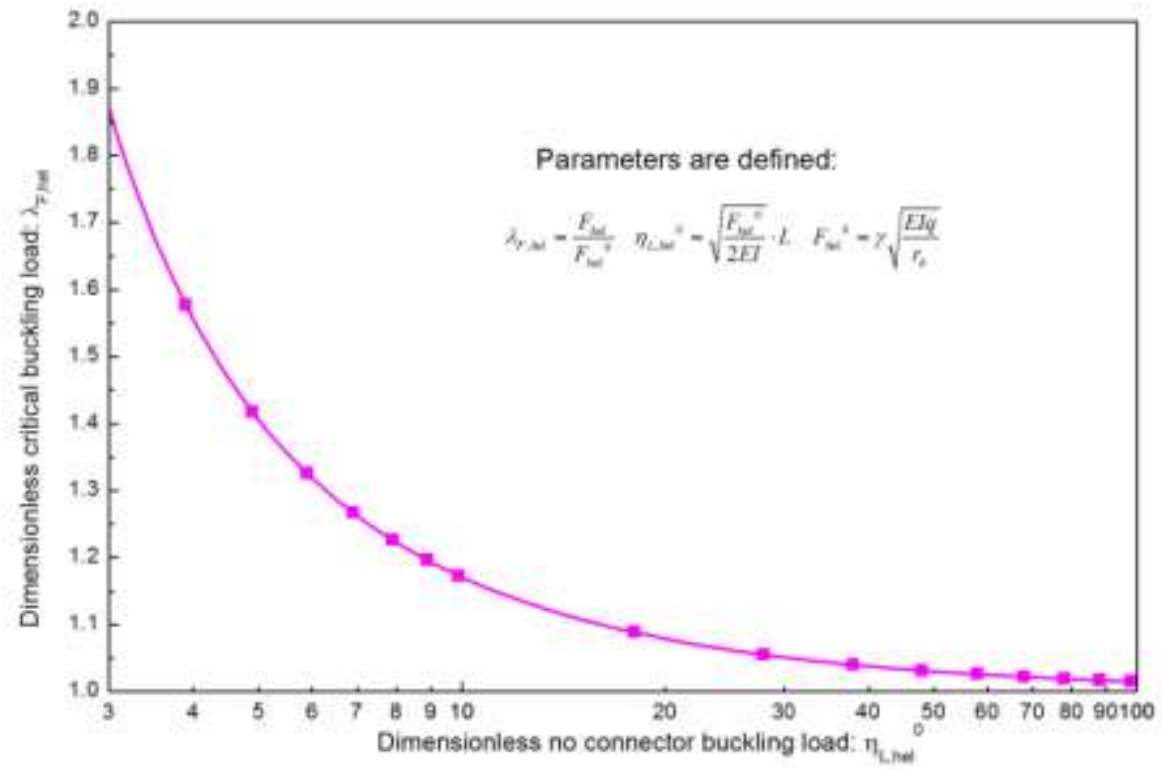

Fig. 7. Critical buckling loads under different values of non-connector critical buckling load.

According to the above studies, the critical inter-helical buckling load $F_{\text {hel,inter }}$ and intra-helical buckling load $F_{\text {hel,intra }}$ are calculated by

$$
\begin{aligned}
& F_{\text {hel, inter }}=\lambda_{\mathrm{F}, \text { hel,inter }} \cdot 2 \sqrt{\frac{2 E I q}{r_{b}}} \\
& F_{\text {hel,intra }}=\left\{\begin{array}{cc}
\max \left(\lambda_{\mathrm{F}, \text { hel,intra }} \cdot 2 \sqrt{\frac{2 E I q}{r_{b}}}, \frac{9.09 \pi^{2} E I}{L^{2}}\right) & \delta_{b} \geq \delta_{\mathrm{b}, \text { intra }} \\
\infty & \delta_{b}<\delta_{\mathrm{b}, \text { intra }}
\end{array}\right.
\end{aligned}
$$

Where $\lambda_{\mathrm{F}, \text { hel,inter }}$ and $\lambda_{\mathrm{F}, \text { hel,intra }}$ are the amendment factors due to connectors and calculated 
by Eq. (6) and (15) respectively. If the axial force is larger than $F_{\text {hel, inter }}$, the inter-helical buckling will occur; if the axial force is larger than $F_{\text {hel, inta }}$, the intra-helical buckling will occur; if the axial force is larger than both $F_{\text {hel,inter }}$ and $F_{\text {hel, intra }}$, the helical buckling mode corresponding to the minimum value of $F_{\text {hel, inter }}$ and $F_{\text {hel,intra }}$ will occur.

\subsection{Contact forces and bending moments}

\section{(1) Contact forces}

The contact force between the tubular string and the wellbore includes that on the connector, first contact point, second point contact and middle continuous contact section. The contact force between the connector and wellbore is calculated by

$$
N_{c}=\frac{1}{2} F \sqrt{\frac{F}{2 E I}} r_{b} \cdot 2 \sqrt{\left(\left.\frac{d^{3} \hat{y}}{d \eta^{3}}\right|_{\eta=0}\right)^{2}+\left(\left.\frac{d^{3} \hat{x}}{d \eta^{3}}\right|_{\eta=0}\right)^{2}}
$$

The contact force on the first contact point $\left(\eta_{1}\right)$ is equal to

$$
N_{p 1}=\frac{1}{2} F \sqrt{\frac{F}{2 E I}} r_{b} \cdot\left[\left(-\left.\frac{d^{3} \hat{y}}{d z^{3}}\right|_{\eta=\eta_{1}^{+}}+\left.\frac{d^{3} \hat{y}}{d z^{3}}\right|_{\eta=\eta_{1}^{-}}\right) \cos \theta_{1}+\left(-\left.\frac{d^{3} \hat{x}}{d z^{3}}\right|_{\eta=\eta_{1}^{+}}+\left.\frac{d^{3} \hat{x}}{d z^{3}}\right|_{\eta=\eta_{1}^{-}}\right) \sin \theta_{1}\right]
$$

The contact force on the second contact point $\left(\eta_{2}\right)$ is calculated by

$$
N_{p 2}=\frac{1}{2} F \sqrt{\frac{F}{2 E I}} r_{b} \cdot\left(-\left.\frac{d^{3} \hat{y}}{d z^{3}}\right|_{\eta=\eta_{1}^{+}}\right)
$$

The contact force on the middle continuous contact section is equal to

$$
N_{w}=\frac{1}{2} F \sqrt{\frac{F}{2 E I}} r_{b} \cdot\left(\eta_{L}-2 \eta_{2}\right)
$$

Then, the total contact force is calculated by

$$
N_{t}=N_{c}+2 N_{p 1}+2 N_{p 2}+N_{w}
$$

The dimensionless total contact force is defined as the ratio of Eq. (23) to the non-connector contact force $N_{t}^{0}=\frac{r_{b} F^{2}}{4 E I}$, namely

$$
\lambda_{n, t}=N_{t} / N_{t}^{0}=1+0.2654 / \eta_{L}
$$

Where $\eta_{L}$ is the dimensionless length of the tubular string and calculated by $\eta_{L}=\sqrt{\frac{F}{2 E I}} \cdot L$.

Note that Eq. (24) is deduced by introducing the deflection curves of intra-helical buckling given in Appendix B into Eq. (23). Thus, the total contact force is calculated by

$$
N_{t}=\lambda_{n, t} \cdot \frac{r_{b} F^{2}}{4 E I}
$$

The ratio of the connector contact force to the total contact force is equal to 


$$
\lambda_{n, c}=\frac{N_{c}}{N_{t}}=\frac{3.4956}{\eta_{L}+0.2654} \text { (26) }
$$

And the ratio of the contact force on tubular string body to the total contact force is equal to

$$
\lambda_{n, b}=1-\frac{N_{c}}{N_{t}}=\frac{\eta_{L}-3.2302}{\eta_{L}+0.2654}(\mathbf{2 7})
$$

Then, the contact forces on connectors and tubular string bodies are calculated with Eq. (12).

\section{(2) Bending moments}

The maximum bending moment on the tubular string is calculated by

$$
M_{\max }=\max _{\eta \in\left[0, \eta_{l}\right]} \frac{F r_{b}}{2} \sqrt{\left(\frac{d^{2} \hat{y}}{d \eta^{2}}\right)^{2}+\left(\frac{d^{2} \hat{x}}{d \eta^{2}}\right)^{2}}
$$

The dimensionless maximum bending moment is defined as the ratio of Eq. (28) to that of the non-connector tubular string under the first category of boundary condition, namely $m_{\max }=M_{\max } /\left(F r_{b} / 2\right) \approx 1.24$. Considering that the maximum bending moment under the secondary category of boundary condition is twice of that under the first category of boundary condition, the maximum bending moment for the connector case under the second category of boundary condition is calculated by

$$
M_{\text {max }}=m_{\max } \cdot F r_{b}
$$

\section{Case studies}

Here the mechanical behaviors of a 5 " drill string constrained in a $12^{1} / 4^{\prime \prime}$ horizontal wellbore are studied. Fig. 8 shows the critical inter-helical buckling and intra-helical buckling loads for different diameters of connectors. The results show that the critical buckling loads increase with the connector diameters in the inter-helical buckling stage and keep constant in the intra-helical buckling stage. Inter-helical buckling tends to occur for the small connector diameter, while intra-helical buckling tends to occur for the large connector diameter. With the increase of the length between adjacent connectors, the critical buckling loads decrease, especially for the larger diameters. Meanwhile, the interval of intra-helical bucking expands with the increase of the length between adjacent connectors. Therefore, increasing the connector diameter and decreasing the length between adjacent connectors can increase the critical helical buckling loads.

Fig. 9 and Fig. 10 respectively show the distributions of contact forces and maximum bending moments in the helical buckling mode. The terms $C_{1}, C_{2}$ and $C_{3}$ respectively represent the non-connector $\left(D_{c}=0.127 \mathrm{~m},\right)$, medium connector $\left(D_{c}=0.219 \mathrm{~m}\right)$ and large connector $\left(D_{c}=0.311 \mathrm{~m}\right)$ cases. Note that $C_{2}$ represents the inter-helical buckling and $C_{3}$ the intra-helical buckling. The results show that the contact forces increase faster and faster and the maximum bending moments increase linearly with the axial force. With the increase of connector diameter, the contact forces and maximum bending moments firstly decrease in the inter-helical buckling stage and then increase in the intra-helical buckling stage. Therefore, intra-helical buckling is more dangerous than inter-helical buckling and should be avoided in the optimal design of 
connectors on tubular strings.

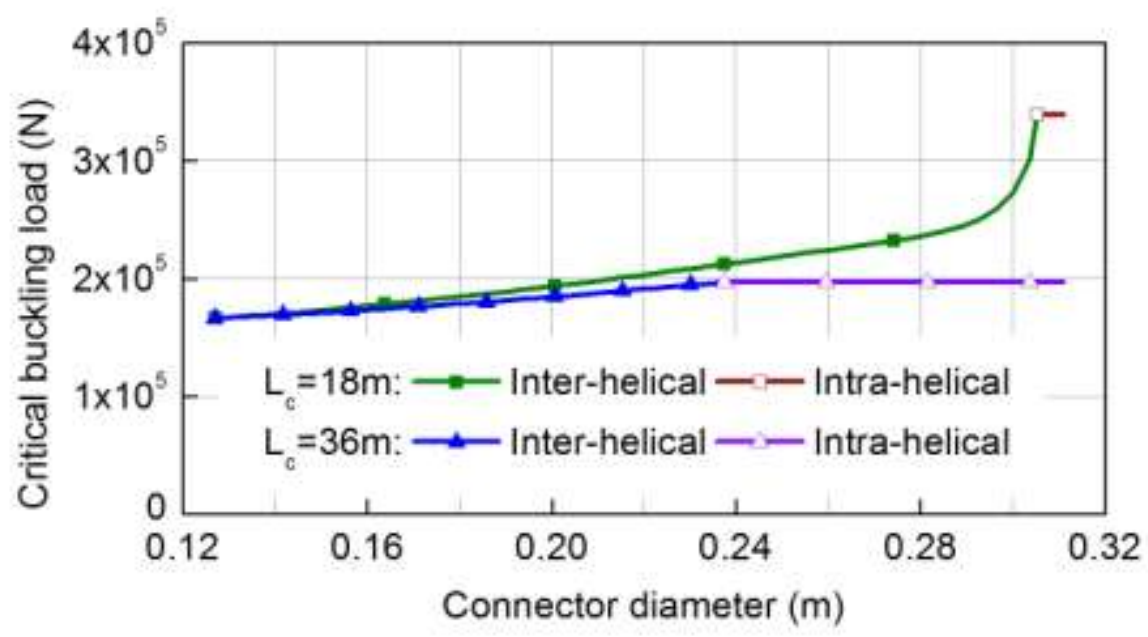

Fig. 8 Critical helical buckling loads for different connector diameters

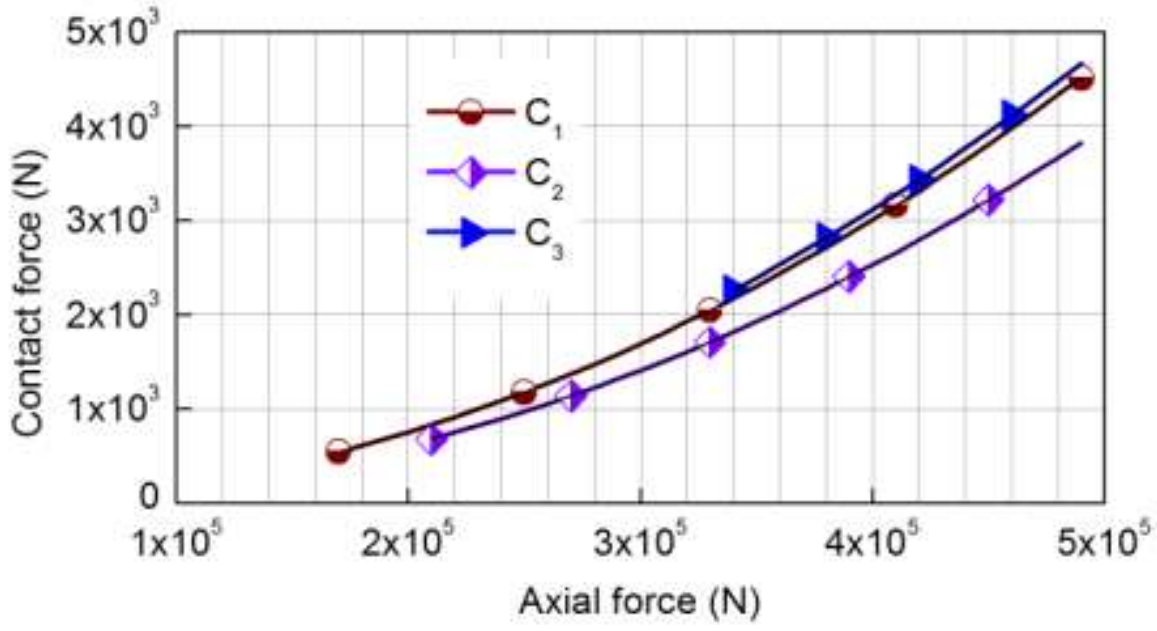

Fig. 9 Contact forces under different axial forces 


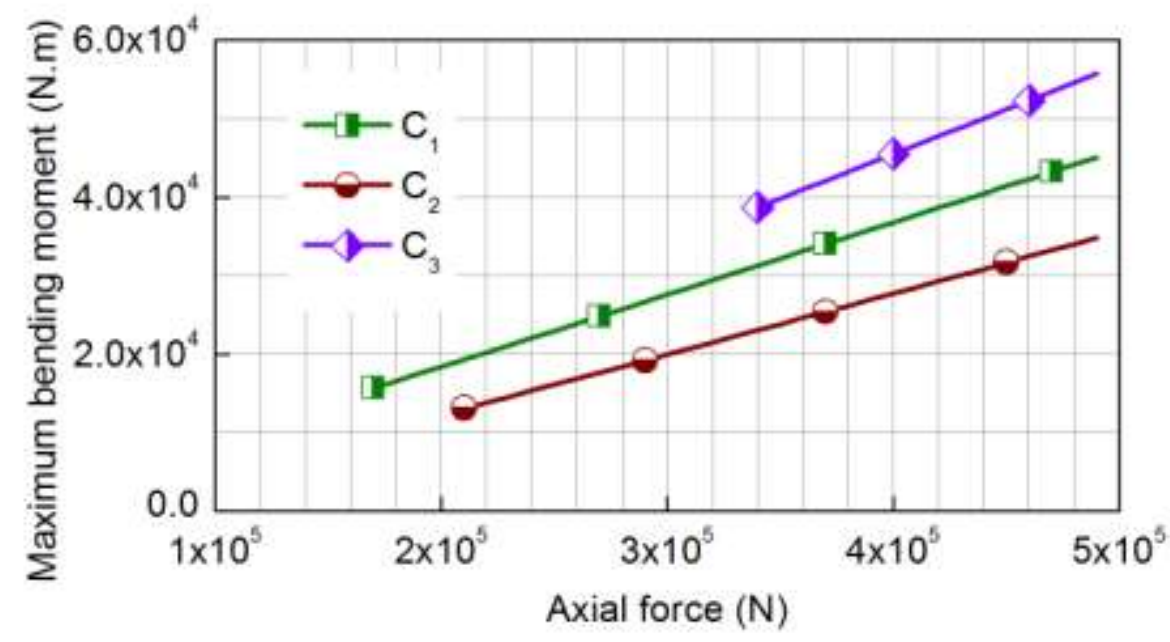

Fig. 10 Maximum bending moments under different axial forces

\section{Further discussions on the effect of connectors}

The effects of connectors are divided into two categories: supporting and constraint effects. The supporting effect means a portion of the tubular string deviates from the inner surface of the wellbore due to the greater size of connector than that of tubular string body. Under the supporting effect, the tubular string tends to move away from the inner surface of the wellbore. The constraint effect means the deflection of the tubular string is affected by the boundary conditions on two adjacent connectors. In another word, the constraint effect makes the deflection of the tubular string between two adjacent connectors more isolated and reduces the interaction effect between adjacent portions of tubular strings (A-B and B-C in Fig. 1).

Generally, these two effects work together in tubular string buckling problem. Taking the inter-helical buckling as example, connectors do not completely isolate the tubular string deflection from adjacent portions of tubular strings but make every portion of the tubular string between two adjacent connectors have the same deflection curve (assumption 4 in section 2). Therefore, the deflection curve of the tubular string with multiple evenly-distributed connectors can be taken as a periodic function with the length between two adjacent connectors as the period. In this case, connectors distribute evenly in the angular direction and the tubular string bodies are seen as the connection elements between adjacent connectors. Therefore, the deflection curve of multiple adjacent segments of the tubular string behaves like an approximate helix. If the diameter of connector is set to be that of the tubular string body, namely $\delta_{b}=1$, the inter-helical buckling reduces to the conventional non-connector buckling problem. With the increase of connector size, the critical contact loads $u_{n p}$ and $u_{p w}$ and critical buckling loads $\lambda_{\text {hel }}$ are all increased due to the supporting effect shown in Fig. 11 and Fig. 2. However, if the diameter of connectors approaches that of the wellbore, the term $\delta_{b}$ and the critical buckling loads $\lambda_{\text {hel }}$ both tend to infinity. Therefore, the inter-helical buckling mode can be thoroughly inhibited by using the "packed" connectors of which the diameter is equal to the wellbore diameter.

However, the intra-helical buckling with packed connectors occurs if the axial compression is large enough. In the intra-helical buckling mode, every tubular string body between adjacent 
connectors separately buckles into helical configuration. Therefore, compared with inter-helical buckling, intra-helical buckling is a higher order buckling mode. The supporting effect is more significant in inter-helical buckling problem, while the boundary effect in intra-helical buckling problem. In another word, the type of the helical buckling mode to occur depends on which effect plays the main role. For the connector of small diameter, the supporting effect is larger than the boundary effect and the tubular string tends to buckle in inter-helical buckling mode. However, for the connector of large diameter, the boundary effect is larger and the tubular string tends to buckle in intra-helical buckling mode.

\section{Conclusions}

1. The helical buckling mode under the presence of connectors on tubular strings is divided into two cases: inter-helical buckling and intra-helical buckling. For connectors of smaller diameters, the supporting effect plays the most important role and inter-helical buckling is easier to initiate. However, for connectors of larger diameters, the constraint effect is dominant and intra-helical buckling is more likely to occur.

2. By introducing the potential energy factor, the critical helical buckling loads for tubular strings with connectors can be obtained by amending the expressions of critical helical buckling loads for non-connector tubular strings. The results indicate that increasing connector diameter and decreasing the length between adjacent connectors can increase critical helical buckling loads.

3. Both the contact forces and maximum bending moments in the intra-helical buckling mode are larger than that in the inter-helical buckling mode. Therefore, intra-helical buckling should be avoided in the optimal design of connectors on tubular strings.

\section{Acknowledgements}

The authors gratefully acknowledge the financial support from the Natural Science Foundation of China (Grant numbers: 51521063), National Science Foundation for Distinguished Young Scholars of China (Grant numbers: 11325211) and China Postdoctoral Science Foundation (Grant numbers: 2016M601044). This research is also supported by national key R\&D projects (Grant numbers: 2016YFC0303303, 2017ZX05009-003).

\section{References}

[1] Lubinski, A. A Study of the Buckling of Rotary Drilling Strings. 1950: American Petroleum Institute.

[2] Lubinski, A. and W.S. Althouse, Helical Buckling of Tubing Sealed in Packers. Journal of Petroleum Technology, 1962. 14(6): 655-670.

[3] Paslay, P.R. and D.B. Bogy, The Stability of a Circular Rod Laterally Constrained to Be in Contact With an Inclined Circular Cylinder. Journal of Applied Mechanics, 1964. 31(4): 605-610.

[4] Mitchell, R.F., Buckling Behavior of Well Tubing: The Packer Effect. Society of Petroleum Engineers Journal, 1982. 22(5): 616-624. 
[5] Mitchell, R.F., New Concepts for Helical Buckling. SPE Drilling Engineering, 1988. 3(3): 303-310.

[6] Mitchell, R.F., A Buckling Criterion for Constant-Curvature Wellbores. SPE Journal, 1999. 4(4): 349-352.

[7] Mitchell, R.F., Exact Analytic Solutions for Pipe Buckling in Vertical and Horizontal Wells. SPE Journal, 2002. 7(4): 373-390.

[8] Chen, Y., Y. Lin and J.B. Cheatham, Tubing and Casing Buckling in Horizontal Wells (includes associated papers 21257 and 21308). SPE Journal of Petroleum Technology, 1990. 42(02): 140-141, 191.

[9] Gao, D., F. Liu and B. Xu. An Analysis of Helical Buckling of Long Tubulars in Horizontal Wells. in SPE International Oil and Gas Conference and Exhibition in China. 1998. Beijing, China: Society of Petroleum Engineers.

[10] Gao, D., Down-Hole Tubular Mechanics and Its Application. 2006, Dongying: China University of Petroleum Press.

[11] Gao, D. and F. Liu, The post-buckling behavior of a tubular string in an inclined wellbore. CMES: Computer Modeling in Engineering \& Sciences, 2013. 90(1): 17-36.

[12] Gao, D., F. Liu and B. Xu, Buckling Behavior of Pipes in Oil \& Gas Wells. Progress in Natural Science, 2002. 12(2): 126-30.

[13] Gao, G. and S. Miska, Effects of Friction on Post-Buckling Behavior and Axial Load Transfer in a Horizontal Well. SPE Journal, 2010. 15(4): 1104-1118.

[14] Gao, G. and S.Z. Miska, Effects of Boundary Conditions and Friction on Static Buckling of Pipe in a Horizontal Well. SPE Journal, 2009. 14(4): 782-796.

[15] Miska, S. and J.C. Cunha. An Analysis of Helical Buckling of Tubulars Subjected to Axial and Torsional Loading in Inclined Wellbores. in SPE Production Operations Symposium. 1995. Oklahoma City, Oklahoma: Society of Petroleum Engineers.

[16] Wu, J., Buckling behavior of pipes in directional and horizontal wells. 1992, Texas A\&M University: Texas.

[17] Wu, J. and H.C. Juvkam-Wold, The Effect of Wellbore Curvature on Tubular Buckling and Lockup. Journal of Energy Resources Technology, 1995. 117(3): 214-218.

[18] Huang, W., D. Gao and F. Liu, Buckling Analysis of Tubular Strings in Horizontal Wells. SPE Journal, 2015a. 20(02): 405 - 416.

[19] Huang, W., D. Gao and S. Wei, Boundary Condition: A Key Factor in Tubular String Buckling. SPE Journal. 2015b. 20(6): 1,409 - 1,420.

[20] Lubinski, A., Fatigue of Range 3 Drill Pipe. Revue de I'Institut Français du Pétrole, 1977. 32(2): 209-232.

[21] Paslay, P.R. and E.P. Cernocky. Bending Stress Magnification in Constant Curvature Doglegs With Impact on Drillstring and Casing. in SPE Annual Technical Conference and Exhibition, 6-9 October, Dallas, Texas. 1991: Society of Petroleum Engineers.

[22] Gao, G., et al., Stability Analysis of Pipe With Connectors in Horizontal Wells. SPE Journal, 2012. 17(3): 931-941.

[23] Huang, W., D. Gao and S. Wei., Local Mechanical Model of Down-hole Tubular Strings Constrained in Curved Wellbores. Journal of Petroleum Science and Engineering. 2015d. 192: 233-242.

[24] Mitchell, R.F., Helical Buckling of Pipe With Connectors in Vertical Wells. SPE Drilling \& 
Completion, 2000. 15(3): 162-166.

[25] Mitchell, R.F., Lateral Buckling of Pipe With Connectors in Horizontal Wells. SPE Journal, 2003a. 8(2): 124-137.

[26] Mitchell, R.F., Lateral Buckling of Pipe With Connectors in Curved Wellbores. SPE Drilling \& Completion, 2003b. 18(1): 22-32.

[27] Mitchell, R.F. and Z.M. Stefan, Helical Buckling of Pipe With Connectors and Torque. SPE Drilling \& Completion, 2006. 21(2): 108-115.

[28] Huang, W. and D. Gao, Sinusoidal buckling of a thin rod with connectors constrained in a cylinder. Journal of Natural Gas Science and Engineering, 2014a. 18(0): 237-246.

[29] Huang, W. and D. Gao, Helical buckling of a thin rod with connectors constrained in a cylinder. International Journal of Mechanical Sciences, 2014b. 84: 189-198.

[30] Huang, W. and D. Gao, Helical buckling of a thin rod with connectors constrained in a torus. International Journal of Mechanical Sciences, 2015c. 98: 14-28.

[31] Huang, W., D. Gao, and Y. Liu, A study of tubular string buckling in vertical wells. International Journal of Mechanical Sciences. 2016,118:231-253.

\section{Appendix-A Inter-helical buckling model}

\section{(1) Basic equations}

According to Huang's work (Huang, 2014b), the lateral deflections of the tubular string between adjacent connectors can be depicted by beam-column model and are calculated by

$$
\begin{aligned}
& y= \begin{cases}\frac{M_{a y}}{F}\left(\frac{\sin k(L-z)}{\sin k L}-\frac{L-z}{L}\right)+\frac{M_{b y}}{F}\left(\frac{\sin k z}{\sin k L}-\frac{z}{L}\right)+r_{c}+\frac{r_{c} \cos \alpha_{A B}-r_{c}}{L} z+\frac{Q_{y} \sin k c}{F k \sin k L} \sin k z-\frac{Q_{y} c}{F L} z & 0 \leq z \leq L-c \text { (30) } \\
\frac{M_{a y}}{F}\left(\frac{\sin k(L-z)}{\sin k L}-\frac{L-z}{L}\right)+\frac{M_{b y}}{F}\left(\frac{\sin k z}{\sin k L}-\frac{z}{L}\right)+r_{c}+\frac{r_{c} \cos \alpha_{A B}-r_{c}}{L} z+\frac{Q_{y} \sin k(L-c)}{F k \sin k L} \sin k(L-z)-\frac{Q_{y}(L-c)(L-z)}{F L} & L-c<z \leq L\end{cases} \\
& x= \begin{cases}-\frac{M_{a x}}{F}\left(\frac{\sin k(L-z)}{\sin k L}-\frac{L-z}{L}\right)-\frac{M_{b x}}{F}\left(\frac{\sin k z}{\sin k L}-\frac{z}{L}\right)+\frac{r_{c} \sin \alpha_{A B}}{L} z-\frac{Q_{x} \sin k c}{F k \sin k L} \sin k z+\frac{Q_{x} c}{F L} z & 0 \leq z \leq L-c \text { (31) } \\
-\frac{M_{a x}}{F}\left(\frac{\sin k(L-z)}{\sin k L}-\frac{L-z}{L}\right)-\frac{M_{b x}}{F}\left(\frac{\sin k z}{\sin k L}-\frac{z}{L}\right)+\frac{r_{c} \sin \alpha_{A B}}{L} z-\frac{Q_{x} \sin k(L-c)}{F k \sin k L} \sin k(L-z)+\frac{Q_{x}(L-c)(L-z)}{F L} & L-c<z \leq L\end{cases}
\end{aligned}
$$

Where $Q_{x}$ and $Q_{y}$ are the contact forces on contact point, $M_{a x}, M_{a y}, M_{b x}$ and $M_{b y}$ are the bending moments at the two connectors, $\mathrm{c}$ is the distance between the right connector and the middle contact point, $\alpha_{A B}$ is the angular displacement between two adjacent connectors, $\mathrm{k}$ is defined by $\sqrt{F / E I}$.

By introducing the following dimensionless parameters,

$$
\begin{array}{ccc}
m_{a y}=M_{a y} \frac{L^{2}}{E I r_{c}} & m_{b y}=M_{b y} \frac{L^{2}}{E I r_{c}} & m_{a x}=M_{a x} \frac{L^{2}}{E I r_{c}} \\
m_{b x}=M_{b x} \frac{L^{2}}{E I r_{c}} & \lambda_{x}=Q_{x} \frac{L^{3}}{E I r_{c}} & \lambda_{y}=Q_{y} \frac{L^{3}}{E I r_{c}} \\
\varepsilon_{c}=\frac{r_{c}}{L} & v=\frac{c}{L} & \lambda_{A B}=\alpha_{A B} / \frac{u}{\sqrt{2}}
\end{array}
$$

We obtain the dimensionless forms of Eq. (30) and (31) as follows 


$$
\begin{gathered}
\hat{y}= \begin{cases}\frac{m_{a y}\left(\frac{\sin u(1-\eta)}{\sin u}-1+\eta\right)+m_{b y}\left(\frac{\sin u \eta}{\sin u}-\eta\right)}{u^{2}}+1+\left(\cos \left(\lambda_{A B} \frac{u}{\sqrt{2}}\right)-1\right) \eta+\frac{\lambda_{y} \sin u v \sin u \eta}{u^{3} \sin u}-\frac{\lambda_{y} v \eta}{u^{2}} & 0 \leq \eta \leq 1-v \\
\frac{m_{a y}\left(\frac{\sin u(1-\eta)}{\sin u}-1+\eta\right)+m_{b y}\left(\frac{\sin u \eta}{\sin u}-\eta\right)}{u^{2}}+1+\left(\cos \left(\lambda_{A B} \frac{u}{\sqrt{2}}\right)-1\right) \eta+\frac{\lambda_{y} \sin u(1-v) \sin u(1-\eta)}{u^{3} \sin u}-\frac{\lambda_{y}(1-v)(1-\eta)}{u^{2}} & 1-v<\eta \leq 1\end{cases} \\
\hat{x}= \begin{cases}-\frac{m_{a x}\left(\frac{\sin u(1-\eta)}{\sin u}-1+\eta\right)+m_{b x}\left(\frac{\sin u \eta}{\sin u}-\eta\right)}{u^{2}}+\sin \left(\lambda_{A B} \frac{u}{\sqrt{2}}\right) \eta-\frac{\lambda_{x} \sin u v \sin u \eta}{u^{3} \sin u}+\frac{\lambda_{x} v \eta}{u^{2}} & 0 \leq \eta \leq 1-v \\
-\frac{m_{a x}\left(\frac{\sin u(1-\eta)}{\sin u}-1+\eta\right)+m_{b x}\left(\frac{\sin u \eta}{\sin u}-\eta\right)}{u^{2}}+\sin \left(\lambda_{A B} \frac{u}{\sqrt{2}}\right) \eta-\frac{\lambda_{x} \sin u(1-v) \sin u(1-\eta)}{u^{3} \sin u}+\frac{\lambda_{x}(1-v)(1-\eta)}{u^{2}} & 1-v<\eta \leq 1\end{cases}
\end{gathered}
$$

The deflection curve of the tubular string is symmetrical with middle point between two adjacent connectors, so the contact force is just on the middle point, namely $v=1 / 2$. Then, Eq. (33) and (34) have 7 unknown terms: $m_{a x}, m_{a y}, m_{b x}, m_{b y}, \lambda_{x}, \lambda_{y}$, and $\lambda_{A B}$. According to the assumption 4 in section 2 , bending moments and rotation angles on the adjacent connectors satisfy periodic conditions, namely

$$
\left[\begin{array}{cccccccc}
0 & -\frac{1}{3} \varepsilon_{c} \psi & 0 & -\frac{1}{6} \varepsilon_{c} \phi & 0 & 1 & 0 & 0 \\
0 & -\frac{1}{6} \varepsilon_{c} \phi & 0 & -\frac{1}{3} \varepsilon_{c} \psi & 0 & 0 & 0 & 1 \\
-\frac{1}{3} \varepsilon_{c} \psi & 0 & -\frac{1}{6} \varepsilon_{c} \phi & 0 & 1 & 0 & 0 & 0 \\
-\frac{1}{6} \varepsilon_{c} \phi & 0 & -\frac{1}{3} \varepsilon_{c} \psi & 0 & 0 & 0 & 1 & 0 \\
\cos \left(\lambda_{A B} \frac{u}{\sqrt{2}}\right) & -\sin \left(\lambda_{A B} \frac{u}{\sqrt{2}}\right) & -1 & 0 & 0 & 0 & 0 & 0 \\
\sin \left(\lambda_{A B} \frac{u}{\sqrt{2}}\right) & \cos \left(\lambda_{A B} \frac{u}{\sqrt{2}}\right) & 0 & -1 & 0 & 0 & 0 \\
0 & 0 & 0 & 0 & -\cos \left(\lambda_{A B} \frac{u}{\sqrt{2}}\right) & \sin \left(\lambda_{A B} \frac{u}{\sqrt{2}}\right) & -1 & 0 \\
0 & 0 & 0 & 0 & -\sin \left(\lambda_{A B} \frac{u}{\sqrt{2}}\right) & -\cos \left(\lambda_{A B} \frac{u}{\sqrt{2}}\right) & 0 & -1
\end{array}\right]\left[\begin{array}{l}
m_{a x} \\
m_{a y} \\
m_{b x} \\
m_{b y} \\
\theta_{a x} \\
\theta_{a y} \\
\theta_{b x} \\
\theta_{b y}
\end{array}\right]=\left[\begin{array}{c}
\frac{1}{u^{2}} \lambda_{y} \varepsilon_{c} \tau_{a}+\varepsilon_{c}\left(\cos \left(\lambda_{A B} \frac{u}{\sqrt{2}}\right)-1\right) \\
\frac{1}{u^{2}} \lambda_{y} \varepsilon_{c} \tau_{b}-\varepsilon_{c}\left(\cos \left(\lambda_{A B} \frac{u}{\sqrt{2}}\right)-1\right) \\
\frac{1}{u^{2}} \lambda_{x} \varepsilon_{c} \tau_{a}-\varepsilon_{c} \sin \left(\lambda_{A B} \frac{u}{\sqrt{2}}\right) \\
\frac{1}{u^{2}} \lambda_{x} \varepsilon_{c} \tau_{b}+\varepsilon_{c} \sin \left(\lambda_{A B} \frac{u}{\sqrt{2}}\right) \\
0 \\
0 \\
0 \\
0
\end{array}\right] \begin{gathered}
\text { (35) } \\
0
\end{gathered}
$$

Where $\theta_{\mathrm{ax}}, \theta_{\mathrm{ay}}, \theta_{\mathrm{bx}}$ and $\theta_{\mathrm{by}}$ are the rotation angles at the connectors, the dimensionless parameters $\tau_{\mathrm{a}}$ and $\tau_{\mathrm{b}}$ are equal to $\tau_{a}=\frac{\sin u v}{\sin u}-v$ and $\tau_{b}=\frac{\sin u(1-v)}{\sin u}$.

To ensure the stability of the helical buckling configuration, the potential energy achieves the minimum value:

$$
\frac{\partial \lambda_{\pi, p}}{\partial \lambda_{A B}}=0
$$

In Huang's work (Huang, 2014b), the energy method is only adopted in the no contact and point contact cases. Here, Eq. (36) is further extended to the wrap contact case.

\section{(2) Contact states}

For the no contact case, the tubular string between two adjacent connectors is not in contact with the wellbore and the contact forces between the tubular string and wellbore are both equal to 0 . Then, the five undetermined variables including $m_{a x}, m_{a y}, m_{b x}, m_{b y}$, and $\lambda_{A B}$ can be calculated with Eq. (35) and (36).

With the increase of axial force, the radial displacement between connectors increases. When the middle point of the tubular string touches the wellbore, the no contact case ends and the point contact is initiated. Therefore, the critical condition between no contact and point contact $u_{n p}$ is obtained by letting 


$$
\max _{\eta=1 / 2} \hat{r}=\delta_{b}
$$

Where $\hat{r}$ is the radial displacement and calculated by $\hat{r}=\sqrt{\hat{x}^{2}+\hat{y}^{2}}$.

For the point contact case, the middle point of the tubular string is in contact with the wellbore and the slope of the radial displacement is tangential to the inner surface of the wellbore at the middle contact point:

$$
\left.\frac{d \hat{r}}{d \eta}\right|_{\eta=1 / 2}=0
$$

Meanwhile, the angular displacement of the concentrated force $\left(\lambda_{x}, \lambda_{y}\right)$ is identical with that of the contact point

$$
\lambda_{x}=\lambda_{m} \sin \theta_{m} \quad \lambda_{y}=-\lambda_{m} \cos \theta_{m}
$$

Where the term $\lambda_{m}$ represents the norm of the dimensionless contact force vector $\left(\lambda_{x}, \lambda_{y}\right)$ at the contact point, and $\theta_{m}$ is the angular displacement of the middle contact point calculated by

$$
\theta_{m}=\frac{u}{2 \sqrt{2}}(\mathbf{4 0 )}
$$

Thus, with Eq. (35), (36), (38) and (39), the 8 undetermined terms including $m_{a x}, m_{a y}, m_{b x}$, $\mathrm{m}_{\mathrm{by}}, \lambda_{\mathrm{x}}, \lambda_{y}, \lambda_{\mathrm{AB}}$ and $\lambda_{\mathrm{c}}$ can be solved.

With the further increase of axial force, the radial displacement between connector and middle contact point increases. The critical condition from point contact to wrap contact $u_{\mathrm{pw}}$ is obtained when the peak of the radial displacement at the middle contact point vanishes, namely

$$
\left.\frac{d^{2} \hat{r}}{d \eta^{2}}\right|_{\eta=1 / 2}=0
$$

Note that the critical no contact to point contact load $u_{n p}$ and the critical point contact to wrap contact load $\mathrm{u}_{\mathrm{pw}}$ are determined by one term: $\delta_{b}$.

For the wrap contact case, the middle portion of the tubular string is in continuous contact with the wellbore. Thus, the tubular string between two adjacent connectors includes two suspended sections near the connectors and one middle continuous contact section. Here we take the two suspended section as a whole, of which the deflection is defined on the region $0 \leq \eta \leq 1-\chi$, while the deflection of the continuous contact section on the region $1-\chi \leq \eta \leq 1$, where $\chi$ represents the ratio of the length of the continuous contact section to that of the tubular string between two adjacent connectors. Here, we assume that the suspended sections be depicted by the buckling configuration in the critical wrap contact case of which the dimensionless tubular string length is set to $(1-\chi)$. Thus, the expressions of the lateral displacements of the suspended sections in the wrap contact case are obtained by respectively replacing $\eta, v$ and $\lambda_{A B}$ by $\frac{\eta}{1-\chi}, \frac{v}{1-\chi}$ and $\lambda_{A B}^{\prime}$ in Eq. (33), (34) and (35). Note that the term $1-\chi$ in the denominator means the coordinate transformation from the region $[0,1]$ to 
$[0,1-\chi]$, and the term $\lambda_{A B}^{\prime}$ means the dimensionless angular displacement change on the suspended sections. Then, the middle point of the suspended sections satisfies:

$$
\left.\frac{d^{2} \hat{r}}{d \eta^{2}}\right|_{\eta=(1-\chi) / 2}=0
$$

For the continuous contact section, the deflection curve of the tubular string is depicted by a helix expressed by

$$
\hat{r}=\delta_{b} \quad \theta=\kappa \cdot \frac{u}{\sqrt{2}} \eta
$$

Note that the change rate of the angular displacement on the continuous contact section is equal to that on the middle contact point of the suspended sections, namely $\kappa \frac{u}{\sqrt{2}}=\left.\frac{d \alpha}{d \eta}\right|_{\eta=\frac{1-\chi}{2}}$.

Then the dimensionless potential energy of (3) is transformed into

$$
\lambda_{\pi, p}=-\frac{4}{u^{4} \delta_{b}^{2}}\left\{\int_{0}^{1-\chi}\left[\left(\frac{\partial^{2} \hat{x}}{\partial \eta^{2}}\right)^{2}+\left(\frac{\partial^{2} \hat{y}}{\partial \eta^{2}}\right)^{2}\right]-u^{2}\left[\left(\frac{\partial \hat{x}}{\partial \eta}\right)^{2}+\left(\frac{\partial \hat{y}}{\partial \eta}\right)^{2}\right] d \eta+\left(\kappa^{4}-2 \kappa^{2}\right) \chi\right\}
$$

The total dimensionless angular displacement change on the suspended sections and continuous contact section is calculated by

$$
\lambda_{A B}=\frac{\lambda_{A B}^{\prime} \frac{u}{\sqrt{2}}+\kappa \cdot \frac{u}{\sqrt{2}} \cdot \chi}{\frac{u}{\sqrt{2}}}=\lambda_{A B}^{\prime}+\kappa \cdot \chi
$$

Where $\lambda_{A B}^{\prime}$ only represent the dimensionless angular displacement change on the suspended sections.

With the above equations, the deflection curve of the tubular string in wrap contact case is determined.

Fig. 11 shows the critical axial forces from no contact to point contact $\left(u_{n p}\right)$ and from point contact to wrap contact $\left(\mathrm{u}_{\mathrm{pw}}\right)$ under different values of the dimensionless radial clearance $\left(\delta_{b}\right)$. The results show that the critical axial forces are rather sensitive to the variation of $\delta_{b}$ when $\delta_{b}$ is close to 1 and tends to stable values with the increase of $\delta_{b}$. When the term $\delta_{b}$ tends to infinity, the critical axial force $u_{n p}$ converges to $\pi$ and $u_{p w}$ to $2 \pi$ respectively. 


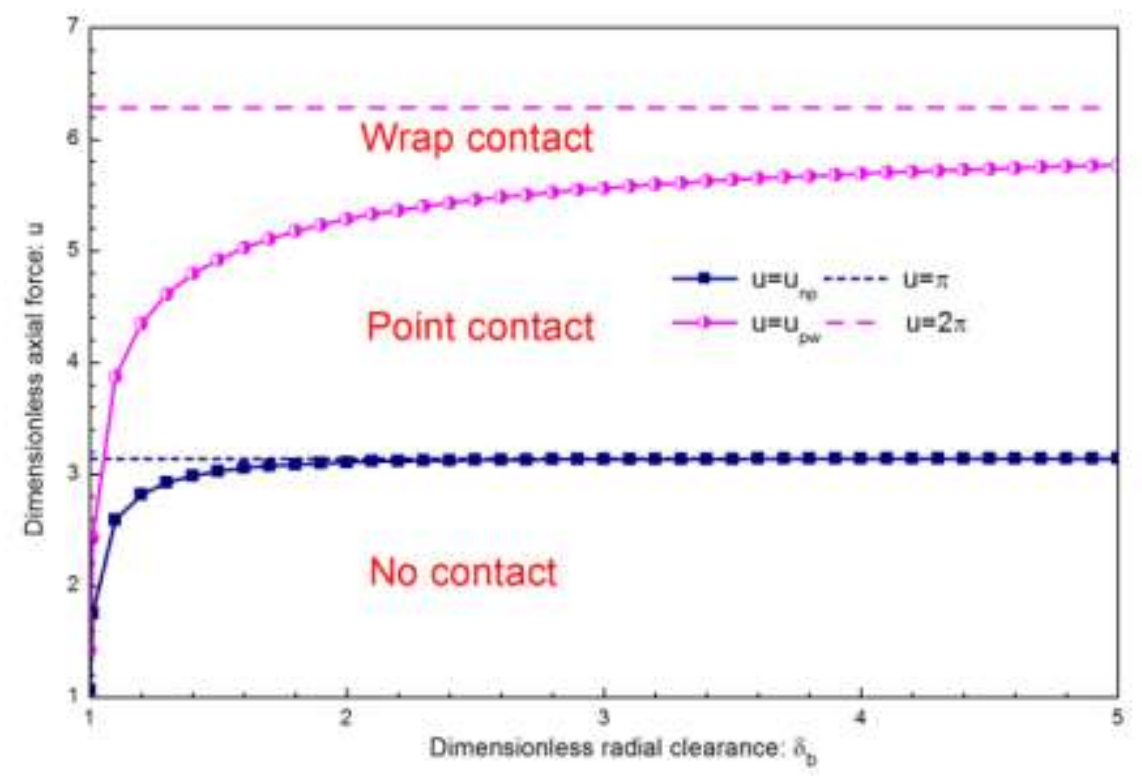

Fig. 11. Contact phase under different values of $u$ and $r_{b} / r_{c}$.

\section{Appendix-B Intra-helical buckling model}

The deflection curve of a tubular string is mainly divided into two parts: (1) suspended sections near the boundary supports and (2) continuous contact section in the middle. The suspended sections on each end of the tubular string are further divided into two suspended portions, each of which is depicted by the beam-column model given by

$$
E I \frac{d^{4} w}{d z^{4}}+F \frac{d^{2} w}{d z^{2}}=0
$$

By introducing the dimensionless distance along the wellbore axis $\eta=\sqrt{\frac{F}{2 E I}} \cdot z$, dimensionless lateral displacements of the tubular string $\hat{y}=\frac{y}{r_{b}}$ and $\hat{x}=\frac{x}{r_{b}}$, and other dimensionless terms in inter-helical buckling, the general solutions of Eq. (46) in two suspended sections are expressed by

$$
\begin{gathered}
\hat{y}_{1}=c_{1} \cos (\sqrt{2} \eta)+c_{2} \sin (\sqrt{2} \eta)+c_{3} \eta+c_{4} \\
\hat{x}_{1}=c_{5} \cos (\sqrt{2} \eta)+c_{6} \sin (\sqrt{2} \eta)+c_{7} \eta+c_{8} \\
\hat{y}_{2}=c_{9} \cos (\sqrt{2} \eta)+c_{10} \sin (\sqrt{2} \eta)+c_{11} \eta+c_{12} \\
\hat{x}_{2}=c_{13} \cos (\sqrt{2} \eta)+c_{14} \sin (\sqrt{2} \eta)+c_{15} \eta+c_{16}
\end{gathered}
$$

Where $\hat{y}_{1}$ and $\hat{x}_{1}$ are the lateral displacements on the first suspended section $\left[0, \eta_{1}\right]$ and $\hat{y}_{2}$ and $\hat{x}_{2}$ are on the second suspended section $\left[\eta_{1}, \eta_{2}\right]$ shown in Fig. $6, c_{1}-c_{16}$ are the 
integral constants. Note that, the tubular string touches the wellbore at the points of $\eta=\eta_{1}$ and $\eta=\eta_{2}$.

For the middle continuous contact section, the deflection curve is assumed to be a helix and expressed by

$$
\begin{aligned}
& \hat{y}_{3}=\cos \left(\eta-\eta_{2}\right) \\
& \hat{x}_{3}=\sin \left(\eta-\eta_{2}\right)
\end{aligned}
$$

According to the symmetry of the deflection curve of the tubular string, only the left half part of the tubular string is considered. The known conditions on the left half portion include:

(1) The lateral displacements and bending moments on the left end $(\eta=0)$ are equal to 0 .

(2) The lateral displacements, tangential directions and bending moments on the first contact point $\left(\eta=\eta_{1}\right)$ are continuous between the first and second suspended sections.

(3) The lateral displacements, tangential directions and bending moments on the second contact point $\left(\eta=\eta_{2}\right)$ are continuous between the second suspended section and the middle continuous contact section.

(4) The deflection of the tubular string is tangent to the inner surface of the wellbore on the first contact point $\left(\eta=\eta_{1}\right)$.

According to the condition (1), we obtain

$$
\begin{array}{cc}
\hat{y}_{1}(0)=0 & \hat{x}_{1}(0)=0 \\
\frac{d^{2} \hat{y}_{1}(0)}{d \eta^{2}}=0 & \frac{d^{2} \hat{x}_{1}(0)}{d \eta^{2}}=0
\end{array}
$$

Assuming that the angular displacement on the first contact point $\left(\eta_{1}\right)$ is $\theta_{1}$, the following expressions are obtained according to the condition (2):

$$
\begin{array}{cl}
\hat{y}_{1}\left(\eta_{1}\right)=\cos \theta_{1} & \hat{x}_{1}\left(\eta_{1}\right)=\sin \theta_{1} \\
\hat{y}_{2}\left(\eta_{1}\right)=\cos \theta_{1} & \hat{x}_{2}\left(\eta_{1}\right)=\sin \theta_{1} \\
\frac{d \hat{y}_{1}\left(\eta_{1}\right)}{d \eta}-\frac{d \hat{y}_{2}\left(\eta_{1}\right)}{d \eta}=0 & \frac{d \hat{x}_{1}\left(\eta_{1}\right)}{d \eta}-\frac{d \hat{x}_{2}\left(\eta_{1}\right)}{d \eta}=0 \\
\frac{d^{2} \hat{y}_{1}\left(\eta_{1}\right)}{d \eta^{2}}-\frac{d^{2} \hat{y}_{2}\left(\eta_{1}\right)}{d \eta^{2}}=0 & \frac{d^{2} \hat{x}_{1}\left(\eta_{1}\right)}{d \eta^{2}}-\frac{d^{2} \hat{x}_{2}\left(\eta_{1}\right)}{d \eta^{2}}=0
\end{array}
$$

Assuming that the angular displacement on the second contact point $\left(\eta_{2}\right)$ is 0 , the following expressions are obtained according to the condition (3):

$$
\begin{array}{cl}
\hat{y}_{2}\left(\eta_{2}\right)=1 \quad \hat{x}_{2}\left(\eta_{2}\right)=0 \\
\frac{d \hat{y}_{2}\left(\eta_{2}\right)}{d \eta}-\frac{d \hat{y}_{3}\left(\eta_{2}\right)}{d \eta}=0 & \frac{d \hat{x}_{2}\left(\eta_{2}\right)}{d \eta}-\frac{d \hat{x}_{3}\left(\eta_{2}\right)}{d \eta}=0 \\
\frac{d^{2} \hat{y}_{2}\left(\eta_{2}\right)}{d \eta^{2}}-\frac{d^{2} \hat{y}_{3}\left(\eta_{2}\right)}{d \eta^{2}}=0 & \frac{d^{2} \hat{x}_{2}\left(\eta_{2}\right)}{d \eta^{2}}-\frac{d^{2} \hat{x}_{3}\left(\eta_{2}\right)}{d \eta^{2}}=0
\end{array}
$$


According to the condition (4), we obtain

$$
\frac{d \hat{y}_{1}\left(\eta_{1}\right)}{d \eta} \cdot \cos \theta_{1}+\frac{d \hat{x}_{1}\left(\eta_{1}\right)}{d \eta} \cdot \sin \theta_{1}=0
$$

From the above analyses, there are 19 unknown parameters including $c_{1}-c_{16}, \eta_{1}, \eta_{2}$ and $\theta_{1}$, and there are also corresponding 19 equations including Eq. (53)-(62). To simplify the calculation, Eq. (53)-(60) are combined into a system of 16 linear equations with respect to $c_{1}-c_{16}$, and then $c_{1}-c_{16}$ are expressed as the functions of $\eta_{1}, \eta_{2}$ and $\theta_{1}$. Further substituting $c_{1}-c_{16}$ into Eq. (61) and (62), three nonlinear equations with respect to $\eta_{1}, \eta_{2}$ and $\theta_{1}$ are obtained. The nonlinear equations can be solved with Newton iteration method and the solutions are expressed as follows:

$$
\eta_{1}=1.696 \quad \eta_{2}=3.349 \quad \theta_{1}=-1.524
$$

And the values of $c_{1}-c_{16}$ are equal to

$$
\begin{array}{llll}
c_{1}=0 & c_{2}=-0.4433 & c_{3}=0.2044 & c_{4}=0 \\
c_{5}=0 & c_{6}=-0.4305 & c_{7}=-0.4172 & c_{8}=0 \\
c_{9}=-0.05339 & c_{10}=-0.5014 & c_{11}=0.09273 & c_{12}=0.1894 \\
c_{13}=0.4042 & c_{14}=0.009834 & c_{15}=0.4281 & c_{16}=-1.4340
\end{array}
$$

Here, we define the critical continuous contact load $\left(\eta_{L, c o n}\right)$ at which the length of the continuous contact section is just equal to 0 . Thus, the critical continuous contact load is expressed by $\eta_{L, c o n}=6.698$ or

$$
\eta_{L, c o n}=2 \eta_{2}=6.698 \quad \text { or } \quad F_{c o n}=\frac{9.09 \pi^{2} E I}{L^{2}}
$$

